Bull. Austral. Math. Soc.

VOL. 39 (1989) [71-80]

\title{
OSCILLATION OF SECOND ORDER NEUTRAL DIFFERENTIAL EQUATIONS
}

\author{
L.H. ERBE AND B.G. ZhaNG
}

\begin{abstract}
Some new sufficient conditions are obtained for the oscillation of the neutral differential equation

$$
\left[r(t)(y(t)-c y(t-\tau))^{\prime}\right]^{\prime}+p(t) y^{\alpha}(t-\sigma(t))=0
$$$$
\text { where } r(t)>0,0<c<1, p(t) \geqslant 0, \sigma(t)>\tau>0 \text { and } \alpha=1 \text { or } 0<\alpha<1 \text {. }
$$

\section{INTRODUCTION}

In the past several years the oscillation problem for second order neutral differential equations of the form

$$
(y(t)+c y(t-\tau))^{\prime \prime}+p y(t-\sigma)=0 \text { where } \tau>0 \text { and } \sigma>0
$$

has been considered by a number of authors [1, 2, 4-8]. Most of these papers treat the case where $c>0$. In $[6,7]$ the case $c<0$ was also studied for Equation (1.1) with constant coefficients and constant delay.

In this paper we consider second order linear and sublinear neutral delay differential equations of the form

$$
\left[r(t)(y(t)-c y(t-\tau))^{\prime}\right]^{\prime}+p(t) y^{\alpha}(t-\sigma(t))=0
$$

where $r, p, \sigma$ are continuous, $r(t)>0,0<c<1,0<\alpha \leqslant 1$ is a quotient of odd integers $\sigma(t)>\tau>0, \sigma^{\prime}(t) \leqslant 1, \lim _{1 \rightarrow \infty}(t-\sigma(t))=\infty$ and $p(t) \geqslant 0$.

As mentioned in [6] there are many important applications for neutral differential equations of the form (1.2).

As usual, a solution of Equation (1.2) is said to be oscillatory if it has arbitrarily large zeros and nonoscillatory if it is eventually positive or eventually negative.

\section{Received 24 March 1988}

Research supported by NSERC- Canada

Copyright Clearance Centre, Inc. Serial-fee code: 0004-9729/89 \$A2.00+0.00. 


\section{LINEAR EQUATIONS}

The following Lemmas will be used to prove the main results.

Lemma 2.1. Assume $0<g(t)<t$ for $t>0, \lim _{t \rightarrow \infty} g(t)=\infty, g \in C\left(\mathbf{R}^{+}, \mathbf{R}^{+}\right)$, $r(t) \in C\left(\mathrm{R}^{+}, \mathrm{R}^{+}\right)$and assume $r(t)$ is either nonincreasing (in brief $\mathrm{n}$.i.) or nondecreasing (in brief n.d.). Let $y \in C\left(\mathbf{R}^{+}, \mathbf{R}\right)$ be such that $r(t) y^{\prime}(t) \in C^{1}\left(\mathbf{R}^{+}, \mathbf{R}\right)$ and $y(t)>0$, $y^{\prime}(t)>0$ and $\left(r(t) y^{\prime}(t)\right)^{\prime} \leqslant 0$ for $t \geqslant T$.

Then for each $0<k<1$ there is a $T_{k} \geqslant T$ such that either

$$
y(g(t)) \geqslant \frac{k r(t) g(t)}{\operatorname{tr}(T)} y(t), \text { for } t \geqslant T_{k} \geqslant T \text { and } r \in n . i .
$$

or

$$
y(g(t)) \geqslant \frac{k g(t)}{t} y(t), \text { for } t \geqslant T_{k} \geqslant T \text { and } r \in \text { n.d. }
$$

Lemma 2.1 is a generalisation of Erbe's Lemma [3]. The proof of this Lemma can be given by the same argument as used in [3] so we omit it here.

LEMMA 2.2. We consider the delay differential inequality

$$
\left(r(t) z^{\prime}(t)\right)^{\prime}-\frac{p(t)}{c} z(t-\sigma(t)+\tau) \leqslant 0
$$

where $r, p, \sigma, c$ and $\tau$ satisfy the assumptions for (1.2) in Section 1. Further assume that either

$$
\limsup _{t \rightarrow \infty} \frac{1}{r(t-\sigma(t)+\tau)} \int_{t-\sigma(t)+\tau}^{t}[u-(t-\sigma(t)+\tau)] p(u) d u>c \text { for } r \in \text { n.i. }
$$

or

$$
\limsup _{t \rightarrow \infty} \frac{1}{r(t)} \int_{t-\sigma(t)+\tau}^{t}[u-(t-\sigma(t)+\tau)] p(u) d u>c \text { for } r \in \text { n.d. }
$$

Then (2.3) has no negative increasing solution.

Proof: Suppose the contrary and let $z(t)$ be a negative increasing solution of (2.3).

Integrating (2.3) we have, for $t>s$

$$
r(t) z^{\prime}(t)-r(s) z^{\prime}(s)-\frac{1}{c} \int_{o}^{t} p(u) z(u-\sigma(u)+\tau) d u \leqslant 0 .
$$


Integrating (2.5) in $s$ from $t-\sigma(t)+\tau$ to $t$, we have

$$
\begin{aligned}
r(t) z^{\prime}(t)(\sigma(t)-\tau) & -\int_{t-\sigma(t)+\tau}^{t} f(s) d z(s) \\
& -\frac{1}{c} \int_{t-\sigma(t)+\tau}^{t}[u-(t-\sigma(t)+\tau)] p(u) z(u-\sigma(u)+\tau) d u \leqslant 0 .
\end{aligned}
$$

We note that $z^{\prime}(t)>0$ so integrating the first integral by parts we have

$$
\begin{aligned}
-r(t) z(t)=r( & -\sigma(t)+\tau) z(t-\sigma(t)+\tau)+\int_{t-\sigma(t)+\tau}^{t} z(s) d r(s) \\
& -\frac{1}{c} \int_{t-\sigma(t)+\tau}^{t}[u-(t-\sigma(t)+\tau)] p(u) z(u-\sigma(u)+\tau) d u \leqslant 0 .
\end{aligned}
$$

For $r \in$ n.d., we have

$$
\int_{t-\sigma(t)+\tau}^{t} z(s) d r(s) \geqslant z(t-\sigma(t)+\tau)[r(t)-r(t-\sigma(t)+\tau)] .
$$

Combining (2.6) and (2.7) we have

$$
\begin{aligned}
& r(t)[z(t-\sigma(t)+\tau)-z(t)]-\frac{1}{c} \int_{t-\sigma(t)+\tau}^{t} \\
& {[u-(t-\sigma(t)+\tau)] p(u) z(u-\sigma(u)+\tau) d u \leqslant 0 . }
\end{aligned}
$$

Dividing the above inequality by $r(t) z(t-\sigma(t)+\tau)$ and noting the negativity of this term, we have

$$
\begin{aligned}
& 1-\frac{z(t)}{z(t-\sigma(t)+\tau)}-\frac{1}{c z(t-\sigma(t)+\tau) r(t)} \int_{t-\sigma(t)+\tau}^{t} \\
& {[u-(t-\sigma(t)+\tau)] p(u) d u \geqslant 0 . }
\end{aligned}
$$

Since $z(t)<0$ and $z^{\prime}(t)>0$, we have

$$
1-\frac{z(t)}{z(t-\sigma(t)+\tau)}-\frac{1}{c r(t)} \int_{t-\sigma(t)+\tau}^{t}[u-(t-\sigma(t)+\tau)] p(u) d u \geqslant 0 .
$$

Hence

$$
\frac{1}{c r(t)} \int_{t-\sigma(t)+r}^{t}[u-(t-\sigma(t)+\tau)] p(u) d u \leqslant 1
$$

which contradicts (2.4).

The case that $r \in n . i$. may be proved in a similar way. We omit the details. 
Remark 2.1. If $p(t) \geqslant p_{0}>0$ and $p_{0}$ is a constant, $r(t) \equiv 1, \sigma(t) \equiv \sigma>\tau$, then (2.3) becomes

$$
z^{\prime \prime}(t)-\frac{1}{c}\left(p_{0}+p(t)-p_{0}\right) z(t-\sigma+\tau) \leqslant 0
$$

By a known result $[\theta$, Theorem 5.3.9], if

$$
\left(\frac{p_{0}}{c}\right)^{1 / 2} \frac{\sigma-\tau}{2}>\frac{1}{e}
$$

then (2.8) has no negative increasing solution.

LEMMA 2.3. In addition to the assumptions for (1.2) in Section 1, further assume that $\sigma(t)$ is nondecreasing and

$$
\liminf _{t \rightarrow \infty} \frac{1}{c} \int_{t-\sigma_{1}(t)}^{t} \frac{1}{r(s)} \int_{0}^{s+\frac{\sigma(\theta)-\tau}{2}} p(u) d u d s>\frac{1}{e}
$$

where $\sigma_{1}(t)=\sigma\left(t+\frac{\sigma(t)-\tau}{2}\right)-\frac{\sigma(t)}{2}-\frac{\tau}{2}$.

Then

$$
z^{\prime}(t)+\frac{1}{c r(t)} \int_{t}^{\infty} p(u) z(u-\sigma(u)+\tau) d u \geqslant 0
$$

has no negative increasing solution.

Proof: If not, let $z(t)$ be a negative increasing solution of (2.11), then

$$
z^{\prime}(t)+\frac{1}{c r(t)} \int_{t}^{t+\frac{(\sigma-\tau)}{2}} p(u) z(u-\sigma(u)+\tau) d u \geqslant 0
$$

By the monotonicity of $z$ we have

$$
z^{\prime}(t)+\left(\frac{1}{c r(t)} \int_{1}^{t+\frac{\sigma-\tau}{2}} p(u) d u\right) z\left(t+\frac{\sigma-\tau}{2}-\sigma\left(t+\frac{\sigma-\tau}{2}\right)+\tau\right) \geqslant 0
$$

By a known result, [8, Theorem 2.1.1], (2.12) has no negative solution under the assumption (2.10). This contradiction proves the Lemma.

In this section we shall henceforth always assume that $\alpha=1$ in (1.2). 
THEOREM 2.1. In addition to the assumption for (1.2) in the first section we assume that $\int_{T}^{\infty} \frac{d t}{r(t)}=\infty$ and either the second order $O D E$

$$
\left(r(t) y^{\prime}(t)\right)^{\prime}+\frac{\lambda p(t) r(t)(t-\sigma(t))}{t r(T)} y(t)=0
$$

is oscillatory for some $0<\lambda<1$ and $r \in$ n.i. or

$$
\left(r(t) y^{\prime}(t)\right)^{\prime}+\lambda p(t) \frac{t-\sigma(t)}{t} y(t)=0
$$

is oscillatory for some $0<\lambda<1$ and $r \in$ n.d. . Then every solution of (1.2) is either oscillatory or tends to zero as $t \rightarrow \infty$.

Proof: Without loss of generality let $y(t)$ be an eventually positive solution of (1.2) and define

$$
z(t)=y(t)-c y(t-\tau)
$$

From (1.2) we know that

$$
\left(r(t) z^{\prime}(t)\right)^{\prime} \leqslant 0 \text { for } t \geqslant T
$$

We shall show that

$$
r(t) z^{\prime}(t)>0 \text { for } t \geqslant T
$$

In fact, if

$$
r(t) z^{\prime}(t)<0 \text { for } t \geqslant T_{1} \geqslant T .
$$

Then

$$
p(t) z^{\prime}(t) \leqslant-\ell<0 \text { for } t \geqslant T_{1} .
$$

Hence

$$
z(t) \rightarrow-\infty \text { as } t \rightarrow \infty,
$$

since $\int_{T}^{\infty} \frac{d t}{r(t)}=\infty$.

On the other hand, if

$$
z(t)<0
$$

we have

$$
0<y(t)<c y(t-\tau)<\ldots<c^{n} y(t-n \tau) .
$$

Hence $y(t) \rightarrow 0$ as $t \rightarrow \infty$, since $0<c<1$. Consequently $z(t) \rightarrow 0$ as $t \rightarrow \infty$ which contradicts the fact that $z(t) \rightarrow-\infty$. Therefore $(2.17)$ is true.

There are two possible cases for $z(t)$ :

(a) $z(t)>0$ for $t \geqslant T_{2} \geqslant T_{1}$,

(b) $z(t)<0$ for $t \geqslant T_{1}$. 
Let us consider the case (a). In this case the assumptions of Lemma 2.1 are satisfied. Therefore for each $0<k<1$ there is a $T_{k} \geqslant T_{2}$ such that

$$
z(t-\sigma(t)) \geqslant \frac{k r(t)(t-\sigma(t))}{r(T) t} z(t), t \geqslant T_{k}, r \in n . i .
$$

and

$$
z(t-\sigma(t)) \geqslant \frac{k(t-\sigma(t))}{t} z(t), t \geqslant T_{k}, r \in n . d . .
$$

Since $0<z(t)<y(t)$, from (1.2) we have

$$
\begin{aligned}
& \left(r(t) z^{\prime}(t)\right)+\frac{k p(t) r(t)(t-\sigma(t))}{t r(T)} z(t) \leqslant 0 \text { for } \lambda<k<1, r \in n . i . ; \\
& \left(r(t) z^{\prime}(t)\right)+k p(t) \frac{(t-\sigma(t))}{t} z(t) \leqslant 0 \text { for } \lambda<k<1, r \in n . d .
\end{aligned}
$$

which imply, respectively that (2.13) and (2.14) are nonoscillatory [3]. This contradicts the assumption.

The second possibility is that $z(t)<0$ for $t \geqslant T$. As before, this time the corresponding solution $y(t)$ must tend to zero as $t \rightarrow \infty$.

THEOREM 2.2. In addition to the assumptions of Theorem 2.1 assume further that (2.4) holds. Then every solution of (1.2) oscillates.

Proof: To prove this theorem it is sufficient to show that in the proof of Theorem $2.1 z(t)<0$, for $t \geqslant T$ is impossible under assumptions (2.4). Suppose that $\left(r z^{\prime}\right)^{\prime} \leqslant 0$, $r z^{\prime}>0$ and $z(t)<0$ for $t \geqslant T$. By (2.15) we have

$$
z(t-\sigma(t)+\tau)>-c y(t-\sigma(t))
$$

This together with (1.2) gives

$$
\left(r(t) z^{\prime}(t)\right)^{\prime}-\frac{p(t)}{c} z(t-\sigma(t)+\tau) \leqslant 0 .
$$

By Lemma $2.2(2.20)$ has no negative increasing solutions which proves the theorem.

TheOREM 2.3. In addition to the assumptions of Theorem 2.1 assume further that $\sigma(t)$ is nondecreasing and (2.10) holds. Then every solution of (1.2) oscillates.

Proof: As mentioned earlier we continue the proof of Theorem 2.1 and consider the possible case that $\left(r z^{\prime}\right)^{\prime} \leqslant 0, r z^{\prime}>0$ and $z(t)<0$ for $t \geqslant T$. From this we have

$$
r(t) z^{\prime}(t) \rightarrow d \geqslant 0
$$


exists. If $d>0$ it follows that $z(t) \rightarrow \infty$ as $t \rightarrow \infty$ which contradicts the negativity of $z(t)$. Therefore $r(t) z^{\prime}(t) \rightarrow 0$ as $t \rightarrow \infty$.

Integrating (1.2) from $t$ to infinity we have

$$
r(t) z^{\prime}(t)=\int_{t}^{\infty} p(s) y(s-\sigma(s)) d s
$$

which, together with $(2.19)$, yields

$$
r(t) z^{\prime}(t) \geqslant-\frac{1}{c} \int_{t}^{\infty} p(s) z(s-\sigma(s)+\tau) d s .
$$

This is a contradiction, by Lemma 2.3. The proof is completed.

Remark 2.2. We consider a special case of (1.2) as follows:

$$
(y(t)-c y(t-\tau))^{\prime \prime}+p(t) y(t-\sigma)=0
$$

where $0<c<1, \sigma>\tau>0$ are constants and $p(t) \geqslant p_{0}>0$. It is obvious (2.13) holds for (2.21). By Remark 2.1 if (2.9) holds then every solution of (2.21) oscillates. Therefore Theorem 8 in [6] becomes a special case of Theorem 2.2.

Example. Consider

$$
(y(t)-c y(t-\pi))^{\prime \prime}+(1+c) y(t-2 \pi)=0
$$

where $0<c<1$. Every solution of (2.22) oscillates by Remark 2.2. In fact $y=\sin t$ is a solution of (2.22).

\section{SUbLINEAR EQUATIONS}

We now consider Equations (1.2) in the sublinear case, that is, $0<\alpha<1$.

Theorem 3.1. Assume that:

(i) the assumptions for (1.2) in Section 1 hold;

(ii) $R(t)=\int_{t_{0}}^{t} \frac{d s}{r(s)}$ and $R(t) \rightarrow \infty$ as $t \rightarrow \infty$;

(iii) every solution of the second order ordinary differential equation

$$
\left(r(t) z^{\prime}(t)\right)^{\prime}+p(t)\left(\frac{\lambda r(t)(t-\sigma(t))}{r(T) t}\right)^{\alpha} z^{\alpha}(t)=0, \text { if } r \in n . i
$$

or

$$
\left(r(t) z^{\prime}(t)\right)^{\prime}+p(t)\left(\frac{\lambda(t-\sigma(t))}{t}\right)^{\alpha} z^{\alpha}(t)=0, \text { if } r \in n . d
$$


is oscillatory, where $0<\lambda<1$ is a constant. Then every solution of (1.2) is either oscillatory or tends to zero as $t \rightarrow \infty$.

Proof: Suppose the contrary and let $y(t)$ be an eventually positive solution. As in the proof of Theorem 2.1 we have $\left(r z^{\prime}\right)^{\prime} \leqslant 0$ and $r z^{\prime}>0$ for $t \geqslant T$. For the case that $z(t)>0$ for $t \geqslant T$, by Lemma 2.1 and (1.2), we get differential inequalities: either

$$
\left(r(t) z^{\prime}(t)\right)^{\prime}+p(t)\left(\frac{k r(t)(t-\sigma(t))}{r(T) t}\right)^{\alpha} z^{\alpha}(t) \leqslant 0
$$

for $t \geqslant T_{k} \quad r \in n . i$. and $0<k<1$ or

$$
\left(t(t) z^{\prime}(t)\right)^{\prime}+p(t)\left(\frac{k(t-\sigma(t))}{t}\right)^{\alpha} z^{\alpha}(t) \leqslant 0 .
$$

By the comparison method we know that (3.3) and (3.4) imply that (3.1) and (3.2) have a nonoscillatory solution [3, p.52], which contradicts assumption (iii). For the case that $z(t)<0$ for $t \geqslant T$, as in the proof of Theorem 2.1 the corresponding solution $y(t)$ tends to zero as $t \rightarrow \infty$. The proof is completed.

Remark 3.1. There are many results for oscillation of second order sublinear ordinary differential equations (3.1) and (3.2). For example, if

$$
\int_{T}^{\infty} R^{\alpha}(t) p(t)\left(\frac{\lambda r(t)(t-\sigma(t))}{r(T) t}\right)^{\alpha} d t=\infty \text { for } r \in n . i
$$

or

$$
\int_{T}^{\infty} R^{\alpha}(t) p(t)\left(\frac{\lambda(t-\sigma(t))}{t}\right)^{\alpha} d t=\infty \text { for } t \in \text { n.d. }
$$

then every solution of (3.1) or (3.2) respectively oscillates.

THEOREM 3.2. In addition to the assumptions of Theorem 3.1 assume further that

$$
\varlimsup_{t \rightarrow \infty} \frac{1}{r(t-\sigma(t)+\tau)} \int_{t-\sigma(t)+\tau}^{t}[u-(t-\sigma(t)+\tau)] p(u) d u>0 \text { for } r \in n . i \text {. }
$$

or

$$
\varlimsup_{t \rightarrow \infty} \frac{1}{r(t)} \int_{t-\sigma(t)+\tau}^{t}[u-(t-\sigma(t)+\tau)] p(u) d u>0 \text { for } r \in n . d .
$$

then every solution of (1.2) oscillates.

Proof: Let $y(t)$ be an eventually positive solution. As in the proof of Theorem 3.1, we have $\left(r z^{\prime}\right)^{\prime} \leqslant 0, r z^{\prime}>0$ and $z(t)<0$ for $t \geqslant T$. From (2.19) and (1.2) we have

$$
\left(r(t) z^{\prime}(t)\right)^{\prime}-\frac{p(t)}{c} z^{\alpha}(t-\sigma(t)+\tau) \leqslant 0 .
$$

By the same arguments as used in the proof of Lemma 2.2 we can prove that (3.9) has no negative increasing solution under assumptions (3.7) and (3.8). Hence we get a contradiction, which porves the theorem. 
TheOREM 3.3. In addition to the assumptions of Theorem 3.1 assume further that $\sigma(t)$ is nondecreasing and

$$
\int_{T}^{\infty} \frac{1}{r(s)} \int_{0}^{a+\frac{(\sigma-\tau)}{K}} P(u) d u d s=\infty
$$

where $K>1$ is some constant. Then every solution of (1.2) oscillates.

Proof: If not, it is sufficient to consider the case that $\left(r z^{\prime}\right)^{\prime} \leqslant 0, r z^{\prime}>0$ and $z(t)<0$ for $t \geqslant T$. As in the proof of Theorem 2.2, we have

$$
\begin{aligned}
r(t) z^{\prime}(t) & =\int_{t}^{\infty} p(s) y^{\alpha}(s-\sigma(s)) d s \\
& \geqslant-\frac{1}{c^{\alpha}} \int_{t}^{\infty} p(s) z^{\alpha}(s-\sigma(s)+\tau) d s \\
& \geqslant-\frac{1}{c^{\alpha}} \int_{t}^{t+\frac{(\sigma-\tau)}{K}} p(s) z^{\alpha}(s-\sigma(s)+\tau) d s \\
& \geqslant-\left(\frac{1}{c^{\alpha}} \int_{t}^{t+\frac{\sigma-\tau}{K}} p(s) d s\right) z^{\alpha}\left(t+\frac{(\sigma-\tau)}{K}-\sigma\left(t+\frac{\sigma-\tau}{K}\right)+\tau\right) .
\end{aligned}
$$

This is a first order sublinear delay differential inequality. From a known result [8, Theorem 3.3.2] (3.11) has no negative solution under assumption (3.10). This contradiction proves the theorem.

Remark. It would be interesting to obtain results similar to those presented here for the superlinear case $\alpha>1$ for equation (1.2).

\section{REFERENCES}

[1] D.D. Bainov and A.I. Zahariev, 'Oscillating properties of the solutions of a class of neutral type functional differential equations', Bull. Austral. Math. Soc. 22 (1980), 365-372.

[2] D.D. Bainov and A.I. Zahariev, 'Oscillating and asymptotic properties of a class of functional differential equations with maxima', Czechoslovak Math. J. 34 (1984), 247-251.

[3] L. Erbe, 'Oscillation criteria for second order nonlinear delay equations', Canad. Math. Bull 10 (1973), 49-56.

[4] S.R. Grace and B.S. Lalli, 'Oscillation of nonlinear second order neutral delay differential equations', Rad. Mat. 3 (1987), 77-84.

[5] M.K. Grammatikopoulos, G. Ladas and A. Meimaridou, 'Oscillations of second order neutral delay diffential equations', Rad. Mat. 1 (1985), 267-274.

[8] M.K. Grammatikopoulos, E.A. Grove and G. Ladas, 'Oscillation and asymptotic behaviour of second order neutral differential equations.', International Conference of Differential Equations at Univ. of Toronto, July 14-16. 
[7] G. Ladas and Y.G. Sficas, 'Oscillations of higher-order neutral equations', J. A ustral. Math. Soc. 27 Ser. B (1986), 502-511.

[8] G. Ladas, E.C. Partheniadis and Y.G. Sficas, 'Necessary and sufficient conditions for oscillation of second order neutral equation', (preprint).

[0] G.S. Ladde, V. Lakshmikanthan and B.G. Zhang, Oscillation theory of differential equations with deviating arguments (Marcel Dekker, New York, Basel, 1987).

Dr L.H. Erbe

Department of Mathematics

University of Alberta

Edinonton, Alberta

Canada T6G 2G1
Dr B.G. Zhang

Shandong College of Oceanography

Qingdao

China 